JENNY MARTEL JACHURA

SANTIAGO, CHILE

MARTELJENNY@UG.UCHILE.CL

ROMINA PACHECO ALARCÓN

SANTIAGO, CHILE

ROMINAPACHECOA@GMAIL.COM

Financiamiento: Proyecto financiado por el

CNCA, bajo el folio 413281

Fecha de recepción: 16/06/2018

Fecha de aceptación: 10/10/2018

Cómo citar: Martel Jachura J.y

Pacheco Alarcón, R. (2018) EntreNiebla:

experimentación de material compuesto,

basado en los desechos de mimbre.

RChD: creación y pensamiento, 3(5), 1-16.

DOI: $10.5354 / 0719-837 \times .2018 .49761$

Revista Chilena de Diseño,

RChD: creación y pensamiento

Universidad de Chile

2018, 3(5)

http://rchd.uchile.cl

\section{EntreNiebla: experimentación de material compuesto, basado en los desechos de mimbre}

\author{
EntreNiebla: experimentation and development of composite \\ material, based on wicker waste
}

Resumen. Proyecto EntreNiebla: Desarrollo de un material compuesto con residuo de mimbre, financiado por el CNCA y el Fondart Nacional 2017, folio 413281 , es un proyecto de investigación que se enmarca en la experimentación de materiales compuestos desarrollados en base a residuos orgánicos, que tiene como finalidad desarrollar un material en base a la fibra de huira de mimbre en desuso, utilizando tecnología de baja complejidad.

Para ello, a. se realizó un levantamiento de información en torno a la cuantificación del residuo de mimbre generado por los artesanos de Chimbarongo, para determinar la capacidad productiva del material; y b. se evaluó el proceso de composición y conformación del material compuesto, para establecer un método procedimental para su conformación y una ficha técnica de sus características principales.

En base a ello, se investigan posibles aplicaciones, en torno a la creación de packaging secundario, para productos elaborados por la Cooperativa de Berries de Chimbarongo, con el fin de otorgar al residuo una vida útil, evitar su incineración y disminuir el impacto ambiental que genera en la comunidad; y a su vez, crear valor agregado en los productos locales, a partir de un empaque que reutilice la materia prima que está siendo desechada.

Palabras clave: material compuesto, residuo de mimbre, reutilización, tecnología de baja complejidad.

Abstract. Proyecto EntreNiebla: Development of a composite material with wicker residue, financed by the CNCA and Fondart 2017, folio 413281; is a research project based on experimentation of composite materials created on the basis of organic waste, which aims to produce a material based on the willow fiber in disuse, using low complexity technology.

To achieve this, a. an information survey carried out to quantify how much wicker residue the Chimbarongo artisans generated. This would allow determining the productive capacity of the material; and $b$. the process of composition and conformation of the composite material was evaluated, to establish a procedural method for its conformation and a technical sheet of its main characteristics.

Based on this results, possible applications were explored around the creation of secondary packaging for products manufactured by the Cooperativa de Berries from Chimbarongo. In order to: give the waste an useful life, avoid its incineration, and reduce the environmental impact that generates in the community; while also creating added value in local products, from a package that reuses raw material that is being discarded.

Keywords: composite material, low complexity technology, reuse, wicker residue 


\section{Introducción}

De un tiempo a esta parte, el estudio en torno a los materiales ha tenido grandes avances. El conocimiento que antes pertenecía exclusivamente a científicos es ahora un ámbito de interés para un público más amplio, lo que permite nuevas posibilidades para el Diseño Industrial (M. Beylerian, 2007). La sostenibilidad, la economía circular y el pensamiento de ciclo de vida han ido conquistando territorios, con el fin de reducir los impactos que generan los procesos productivos, por los cuales se conforma nuestra cultura material y entorno. Bajo esta premisa, se visualiza un incremento en la producción de materiales en base a residuos de procesos industriales o artesanales que han ampliado la gama de materiales compuestos y permiten reemplazar o ser una alternativa a los materiales tradicionalmente usados.

Por otra parte, el mimbre es un producto forestal no maderero, de gran significación social, ya que permite una fuerte incorporación de mano de obra en comunidades rurales. En el país, el cultivo y la producción en torno al mimbre se concentra en la comuna de Chimbarongo, lo cual genera oportunidades de trabajo para artesanos y trabajadores de la provincia de Colchagua. Sin embargo, la actividad artesanal que caracteriza esta zona no está exenta de impacto ambiental y social, ya que la obtención de la huira genera un residuo que en la actualidad no es gestionado, y tiene como fin de vida la incineración.

Bajo este contexto, nace Proyecto EntreNiebla como proyecto de investigación de carácter experimental, el que busca optimizar los porcentajes de aprovechamiento de la materia prima de la artesanía del mimbre mediante la reintegración del residuo en la economía y el desarrollo de productos que puedan integrarse en la oferta local de la comuna de Chimbarongo, y donde puedan proyectarse nuevas posibilidades para su aplicación y uso en el ámbito del Diseño Industrial.

\section{Estrategia metodológica}

Debido a que la información sobre el mimbre es escasa, se realiza una primera etapa exploratoria. Para ello se desarrolla una investigación de campo y revisión bibliográfica, la que consistió en: (a.) obtener información cuantitativa sobre el volumen del residuo, (b.) obtener información cualitativa sobre el impacto generado por el residuo y (c.) determinar la escala de producción a partir de esa información.

Además, se realiza una revisión bibliográfica referente a los materiales compuestos biobasados, la que permite delimitar (a.) los instrumentos y criterios de selección de los componentes a utilizar, y (b.) las estrategias de experimentación.

Por otra parte, se realiza una etapa experimental que se lleva a cabo mediante la realización de experimentos para la evaluación de la composición y conformación del material, con el fin de establecer un método procedimental y una ficha técnica de sus características principales.

Por último, se lleva a cabo una etapa de validación para determinar la aplicación del material. Para ello, se realiza (a.) una investigación sobre tipología de packaging secundario de productos derivados de la producción de berries (mermeladas, siropes, entre otros), y (b.) un trabajo colaborativo con la presidenta de la Cooperativa de Berries, Ana Córdova, quien proporciona información en torno a la venta de sus productos. A partir de ello, se obtienen insights para la elaboración de una serie corta del packaging, expuestos en la Expo Mimbre 2018. 
2. Hipótesis

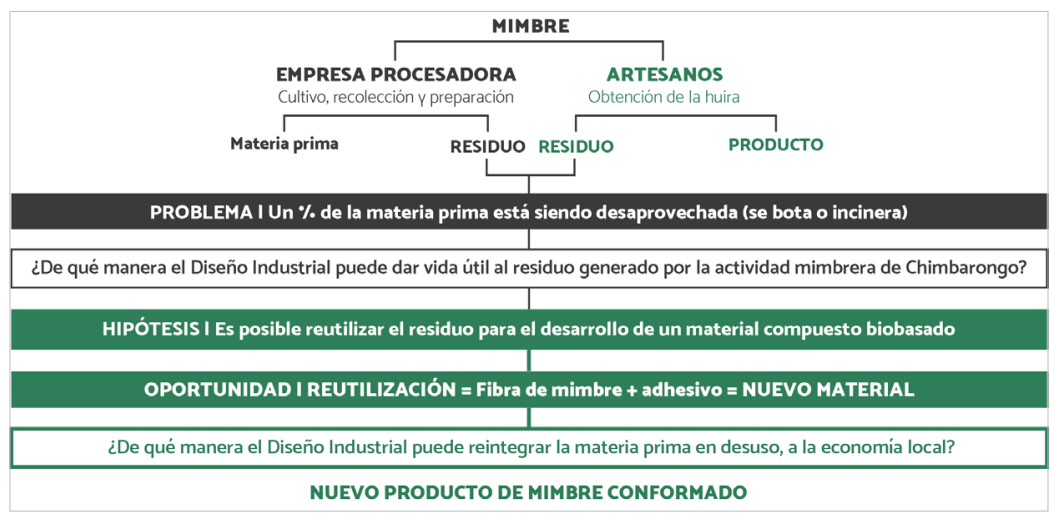

\section{Objetivos}

El objetivo general del proyecto es desarrollar y caracterizar un nuevo material compuesto, en base al residuo de huira de mimbre y un aglutinante natural, para extender su vida útil, a través de la aplicación en packaging secundario de productos de elaboración local de Chimbarongo.

Para ello, los objetivos específicos son: 1. cuantificar el residuo mensual de huira de mimbre en la localidad de Chimbarongo para establecer parámetros de capacidad productiva del material; 2 . definir el proceso de composición y conformación del material compuesto, y caracterizar sus propiedades para desarrollar un material que rescate y reduzca los residuos generados por la actividad mimbrera; y 3 . evaluar el uso del material compuesto, por medio de su aplicación en el desarrollo de packaging secundario para validar la extensión de la vida útil del residuo rescatado.

\section{Marco teórico: teoría y práctica sobre la conformación de un material biocompuesto \\ 4.1. Bio composites}

Dada la creciente preocupación hacia el uso de tecnologías más sustentables, los materiales compuestos derivados de fuentes naturales y renovables han ganado interés en los últimos años. En muchos casos este tipo de materiales biobasados ofrecen mejor funcionalidad, menor peso y beneficios respecto a la salud. En cuanto a los beneficios medioambientales de las fuentes biobasadas, se encuentra el bajo consumo energético, disminución de emisión de $\mathrm{CO} 2$, reducción en el consumo de combustibles fósiles y un impacto positivo en la agricultura. En los últimos años, las combinaciones de las fibras naturales y polímeros biobasados han demostrado tener propiedades de materiales compuestos, lo que ofrece la atractiva perspectiva de que los compuestos totalmente biobasados son una realidad comercial en aumento (Biocomposites: Technology overview. Marzo 2014. s.f.).

Las fibras naturales como el lino, cáñamo, yute, entre otras tienen buena resistencia y rigidez, a su vez son mucho más livianas que los materiales de refuerzo convencionales como la fibra de vidrio; tienen un costo relativamente bajo y son biodegradables. En la actualidad, estas fibras se utilizan en cantidades importantes, específicamente en componentes automotrices, como refuerzo de polímeros sintéticos como el polipropileno (PP) (Biocomposites: Technology overview. Marzo 2014. s.f.).
Esquema 1. Hipótesis de investigación. 
Por otra parte, el desarrollo de polímeros de fuentes renovables ha recibido bastante atención, debido a la volatilidad de los precios del petróleo y el deseo de evitar la eliminación de desechos en vertederos. A partir de ello, se han desarrollados polímeros termoplásticos y termoestables en base a productos químicos biológicos, donde las principales fuentes han sido los aceites de plantas y almidones (Biocomposites: Technology overview. Marzo 2014. s.f.).

\subsection{Resinas termoestables}

\subsubsection{Aceites vegetales}

Una amplia gama de aceites de plantas se puede convertir en resina termoestable. Los aceites vegetales son triglicéridos que pueden ser funcionalizados con grupos reactivos como hidroxilo, carboxilo, amida y epoxi, y, por lo tanto, pueden ser reticulados para formar polímeros rígidos.

Los estudios acerca de este tipo de resinas se han desarrollado principalmente en países del hemisferio norte, como en Estados Unidos que ha trabajado en torno a resinas en base a soya; y Reino Unido que ha avanzado en la creación de resinas más estables al calor. Por otra parte, se han desarrollado estudios en torno al líquido de la cáscara de cajú, que puede convertirse en resinas termoestables, y que tienen su desarrollo en Estados Unidos, Reino Unido e India.

\subsubsection{Alcohol poli furfurílico (PVA)}

Las resinas de alcohol polifurfurílico (PVA) son una familia derivada del alcohol furfurílico, un alcohol renovable producido del furfural que se forma por la digestión catalizada con ácido de los azúcares hemicelulósicos en la biomasa. La materia prima apropiada son los desperdicios de agricultura, el más común es el bagazo de caña de azúcar. Las propiedades de estas resinas, que incluye niveles de estabilidad térmica, resistencia química y retardantes de fuego, son particularmente atractivas para la industria de los compuestos.

\subsection{Materiales compuestos basados en maderas}

Según Stark (2010), se llama así a cualquier material lignocelulósico aglutinado con una resina. Los tableros de partículas son el más común en aplicaciones interiores de este tipo de material, y utiliza hasta $10 \%-15 \%$ de adhesivo de urea formaldehído (UF). Otro tipo de compuestos con base maderera son los compuestos de fibra vegetal, que contienen hasta $60 \%$ de madera, contenidos en una matriz termoplástica, termoestable o elastomérica (Wechsler, 2014, p. 7).

La investigación de Andrea Wechsler (2014), plantea el término "tablero de partículas" para cualquier compuesto lignocelulósico unido con $20 \%$ o menos de adhesivo. El término adhesivo se refiere a una resina termoestable utilizada para pegar partículas. El término compuesto se usa para el resto de los compuestos lignocelulósicos con $40 \%$ de matriz. La matriz que se usa para unir los compuestos se refiere a una resina termoplástica o termoestable. Por lo tanto, la diferencia radica en el tipo de resina que se utiliza para unir las partículas lignocelulósicas. Por otra parte, Baillie (2004) establece que los rellenos de fibras naturales pueden definirse como materiales celulósicos. Estos, son la forma de biomasa más abundante y la más probable de ser usada como refuerzo en compuestos de madera, no solo por razones ecológicas y económicas, sino que también por su alto desempeño mecánico y térmico, bajo costo y disponibilidad (Wechsler, 2014, p. 17). 
Çöpür et al. (2007) menciona en su estudio que desde el 1900 se han utilizados derivados de agroindustrias como materia prima para la fabricación de compuestos de madera, lo que plantea un futuro prometedor para los tableros fabricados con fuentes alternativas. Sin embargo, uno de los problemas relacionados a la utilización de estas fibras es el alto costo de recolección, transporte y almacenamiento. Esto se podría resolver acercando las industrias que fabrican dichos tableros a las áreas rurales de producción, donde se generan los residuos (Wechsler, 2014, p. 17).

Wool and Sun (2005) establece que la propiedad de los compuestos fabricados con derivados de agroindustrias depende de muchas variables, como la localidad de la planta, la sección utilizada de la planta, el método de su cosecha y cómo esta es precondicionada. Las fibras naturales no son de propiedades uniformes, pero la ventaja de estas fibras es que cualquiera se puede usar para hacer compuestos de bajo costo (Wechsler, 2014, p. 17).

\subsection{Proceso de conformación de materiales compuestos Moldeo por compresión}

En el moldeo por compresión, el material líquido o en forma de masa se introduce en el molde y este se cierra hasta que las dos mitades del molde ejercen presión en el material. Conforme el molde va cerrándose, el material es obligado a ocupar todas las partes de la cavidad del molde. En ciertos casos, es ventajoso realizar el moldeo cerrando primeramente el molde casi por completo y abriéndolo después unos segundos antes de aplicar la presión definitiva. De esta forma, se deja "respirar" al material para permitir la evacuación del gas que queda atrapado (Beltrán y Marcilla, 2012).

Una vez que el molde se ha cerrado completamente, se aplica la máxima presión que provoca el llenado final de la cavidad. Bajo la acción de presión tiene lugar la reacción de entrecruzamiento que transforma al material termoendurecible en termoestable, proceso que se conoce vulgarmente como "curado". Tras el curado se abre el molde y se extrae la pieza totalmente sólida, la que alcanza su rigidez definitiva, por lo general, después de 24 horas (Beltrán y Marcilla, 2012).

\section{Levantamiento de información: aproximaciones en torno al residuo generado por la actividad artesanal del mimbre de Chimbarongo}

\subsection{Descripción}

Esta etapa consistió en la recopilación de datos sobre el residuo de huira de mimbre generado por los artesanos de Chimbarongo, con el fin de obtener información que permita determinar la cantidad de residuo desechado y la capacidad productiva del material.
Esquema 2. Ventajas y limitaciones del moldeo por compresión. Fuente: Beltrán y Marcilla, 2012.

\section{MOLDEO POR COMPRESIÓN}

VENTAJAS

a. Esta técnica no genera recortes

b. Bajas cantidades de mermas

c. Es posible fabricar piezas de sección fina

d. Los moldes y la maquinaria son más económicos

\section{LIMITACIONES}

a. No se pueden realizar formas complejas

b. Para conseguir que el molde se llene completamente con un material de viscosidad elevada es necesario emplear presiones elevadas 
Gráfico 1 y 2. Tipología de residuo generado y Formas de desechar el residuo.

Para ello, se realizó una investigación de tipo exploratoria, donde los instrumentos utilizados corresponden a fuentes de información primaria obtenida de documentación municipal, tesis universitarias, encuestas, entrevistas y estudio de campo.

\subsection{Universo de artesanos de Chimbarongo}

Se levanta información referente al número de artesanos mimbreros de Chimbarongo, debido a que no existen datos formales que determinen su universo total. Para ello, se contrastan cinco listados proporcionados por la Municipalidad de Chimbarongo, que corresponden a los participantes de la Expo Mimbre en sus distintas versiones. Del contraste, se genera la primera base de datos, en la cual se identifican 112 artesanos.

\subsection{Residuo de huira de mimbre}

De forma paralela, se realiza una revisión bibliográfica en torno al residuo que genera la actividad artesanal. Para ello, se revisa la tesis "Rediseño del partidor de la vara de mimbre", que tiene como objetivo optimizar el rendimiento de la vara partida, y con ello lograr que el proceso de obtención de la huira mejore (Muñoz, Guillermo, 2016).

De ello, se rescatan datos sobre la cantidad de residuo generado en el proceso de partido y obtención de la huira, donde el autor determina que de un paquete de mimbre que pesa 10.000 gr., se pierde un $68 \%$ de la materia en el proceso de descostillado, medulado y rebajado.

\subsection{Encuesta artesanos}

Posteriormente, se aplica una encuesta a una muestra aleatoria de setenta y dos artesanos, la que busca obtener información sobre: 1. su quehacer, para identificar la tipología de productos presente en la localidad; 2. su aprendizaje, para identificar el traspaso de la técnica; y 3 . su residuo, para medir la cantidad aproximada de acumulación en el período de una semana. Con esta herramienta, se identifica que el $94 \%$ de los artesanos encuestados genera residuo denominado huira falsa, el cual se obtiene del proceso de desmedulado y rebajado. Por otra parte, un $71,4 \%$ se deshace del residuo quemándolo en su patio o en sectores eriazos, dato que permite visualizar el impacto ambiental del residuo generado por la combustión de la fibra, que se traduce en un aumento de partículas en suspensión.

\subsection{Estudio de campo}

Finalmente, se ejecuta una versión piloto de la gestión de residuo, donde se realiza un seguimiento a nueve artesanos, seleccionados a partir del análisis

¿Cuál es el residuo que más genera?

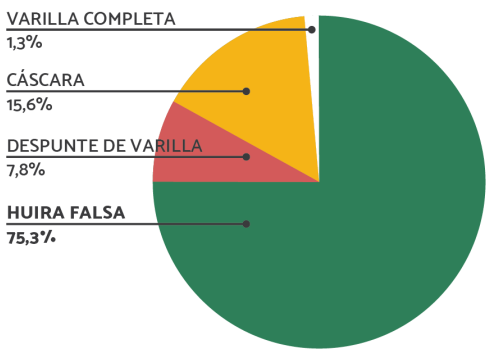

¿Qué forma de desechar el residuo utiliza con mayor frecuencia?

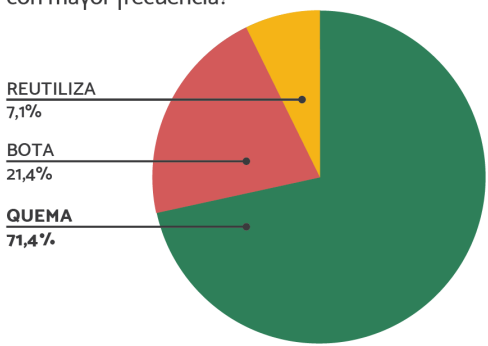


de la encuesta, durante el período de un mes. Estos representan las categorías de generación de residuo: baja, media y alta.

De esta actividad se desprende lo siguiente:

a. El peso y volumen del residuo varía de acuerdo a la técnica del artesano. Las técnicas de la artesanía del mimbre son diversas, lo que incide en la cantidad y calidad del residuo que genera cada artesano. Por una parte, hay artesanos que trabajan con huira fina, como Segundo Rodríguez, donde el residuo es una viruta de gran volumen y poco peso; mientras que otros realizan un trabajo rústico, como Sergio Vergara, en el que su residuo es de poco volumen y mucho peso.

b. La cantidad de residuo generado varía de acuerdo a la temporada. El estudio de campo permitió identificar temporadas de trabajo de la actividad artesanal. Este se ejecutó en temporada media, por lo que los resultados deben extrapolarse a las otras dos temporadas para determinar el volumen del residuo anual.

\subsection{Justificación}

\subsubsection{Selección de material}

Tipo de residuo. Se selecciona el residuo generado por el proceso de desmedulado y rebajado de la huira de mimbre. Esto se basa en la calidad del residuo que se obtiene en esta etapa, ya que proviene de la varilla de mimbre, la que ha sido previamente procesada y seleccionada, y por lo tanto, cumple con normativas de calidad.
Tabla 1. Relación tipo de huira - peso de residuo.

Esquema 3. Temporadas de actividad artesanal en Chimbarongo.

\begin{tabular}{ccccc} 
ARTESANO & TIPO HUIRA & $\begin{array}{c}\text { PESO MIN X } \\
\text { SACO (kg) }\end{array}$ & $\begin{array}{c}\text { PESO MÁX. X } \\
\text { SACO (kg) }\end{array}$ & PESO PROM. \\
\hline Segundo Rodríguez & Fina & 1,85 & 3 & 2,4 \\
\hdashline Rodolfo Castro & Media & 3,52 & 5,24 & 4,4 \\
\hdashline Sergio Vergara & Gruesa & 4,70 & 6,55 & 5,6
\end{tabular}

TEMPORADA ACTIVIDAD ARTESANAL

ALTA

noviembre

diciembre

enero

febrero

marzo
MEDIA

octubre
BAJA

abril

mayo

junio

julio

agosto 
Tabla 2. Peso total del residuo del mes de octubre - temporada media.

Fuente: elaboración propia

Figura 1. Tipos de residuos.

Fuente: elaboración propia.

Esquema 4. Etapas de experimentación.

\begin{tabular}{|c|c|c|c|c|}
\hline ARTESANO & $\begin{array}{l}\text { CATEGORÍA } \\
\text { GENERACIÓN } \\
\text { DE RESIDUO }\end{array}$ & $\begin{array}{l}\text { BOLSAS POR SEMANA } \\
\text { (DATOS ESTUDIO DE } \\
\text { CAMPO) }\end{array}$ & $\begin{array}{c}\text { PESO PROM. BOLSAS } \\
\text { (kg) (DATO ESTUDIO } \\
\text { DE CAMPO) }\end{array}$ & $\begin{array}{l}\text { PESO TOTAL } \\
\text { DEL MES (kg) }\end{array}$ \\
\hline Hns. Torrealba & Alta & 8 & 1,62 & 51,8 \\
\hline Sergio Vergara & Alta & 7 & 5,79 & 162,1 \\
\hline Miguel Ortega & Alta & 3 & 4,16 & 49,9 \\
\hline Fredy Jorquera & Media & 2 & 2,09 & 16,72 \\
\hline Rodolfo Castro & Media & 8 & 3,35 & 107,2 \\
\hline Segundo Rodríguez & Baja & 2 & 1,94 & 15,5 \\
\hline Félix Toledo & Baja & 3 & 3,66 & 43,9 \\
\hline Claudio Lorca & Baja & 3 & 2,1 & 25,2 \\
\hline Carlos Pino & Baja & 1 & 1,05 & 4,2 \\
\hline Total & & & & 476,52 \\
\hline
\end{tabular}

\subsection{Tipologías de residuos}

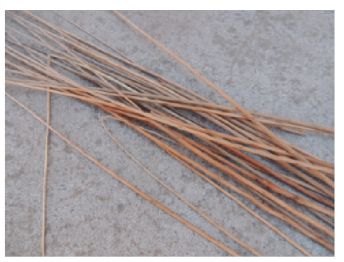

Despunte de Varilla Resultado del cortar de los extremos más delgados e irregulares de la varilla de mimbre.

Residuo de volumen y peso medio.

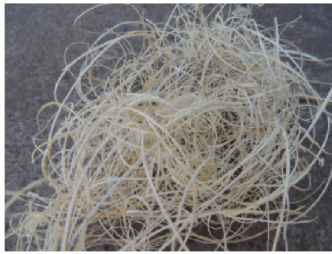

Descostillado

Resultado de rebajar reiteradamente la huira con la que va a trabajar, para obtener trabajos más finos.

Residuo de gran volumen y poco peso.

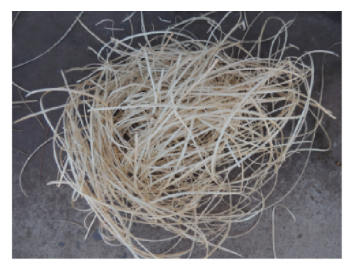

Descorazonado

Resultado de partir la varilla en 4 partes, $y$ extraer su parte central. Residuo de mucho volumen y poco peso.

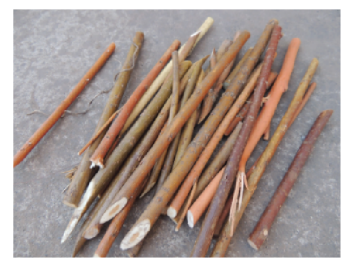

Despunte de Varilla

Resultado de cortar las varillas una vez que el producto está listo, para eliminar los excedentes.

Residuo de poco volumen y mucho peso.

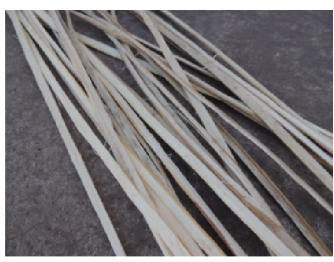

Huira Falsa

Resultado de pasar las partes de la varilla partida, por la rebajadora, para obtener la huira con las que se trabajará.

Residuo de gran volumen y peso medio.

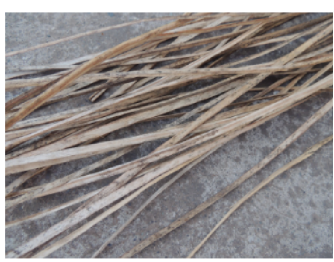

Varilla completa

El residuo de varilla completa se produce cuando la varilla se deja remojar durante mucho tiempo y se pudre.

Es un residuo de gran volumen, de gran peso $y$ húmedo.

\section{Experimentación: estudio de la conformación de un material biocom- puesto en base a fibra de mimbre}

\section{ETAPA 1: COMPOSICIÓN DEL MATERIAL ETAPA 2: CONFORMACIÓN DEL MATERIAL}

Explorar y evaluar el proceso de composición de un material compuesto realizado en base a residuo de huira de mimbre y un adhesivo.

Actividades

1. Análisis comparativo de los adhesivos

2. Análisis comparativos de las características organolépticas
Evaluar el proceso de conformación y mecanizado del material compuesto, $y$ sus propiedades mecánicas.

Actividades

1. Ensayo mecánico del material.

2. Experimentación de moldeabilidad y mecanizado 

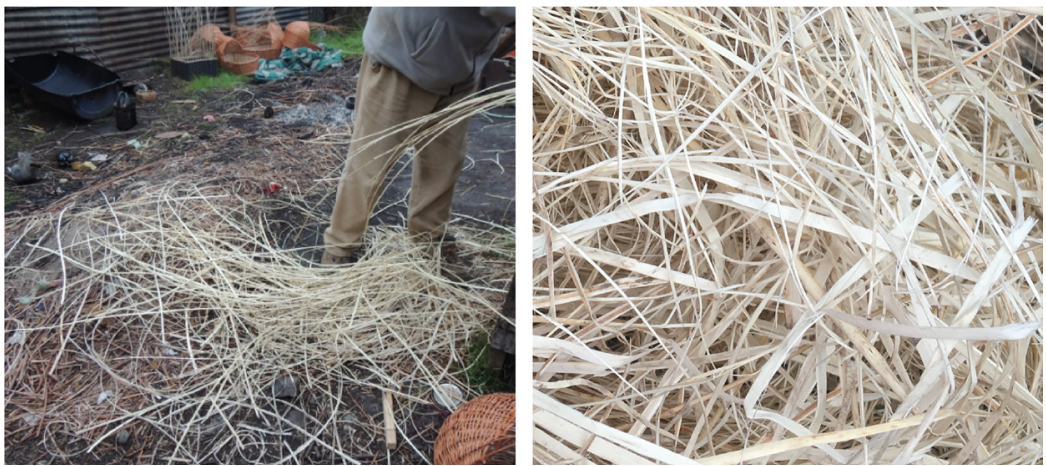

6.1.2. Tipo de adhesivo. Se seleccionan cinco tipos de adhesivos para experimentar la composición del material, de acuerdo a la revisión bibliográfica y la factibilidad de su adquisición para el proyecto: PU Khel, PU espuma Khel, Adhesivo acuoso Graflex (lote muestra $n^{\circ} 3$ ), PU Graflex (PM-100), PVA.

\section{Se trabaja con estos adhesivos, teniendo en consideración:}

a. Origen del adhesivo: lugar de producción local (Chile) y/o regional (Latinoamérica).

b. Proceso productivo para generar el material: tiempo y temperatura de curado, precauciones en el proceso de producción, costo del adhesivo.

c. Uso: tiempo de vida útil.

\subsection{Criterios a evaluar}

Para el objetivo específico 1: composición del material

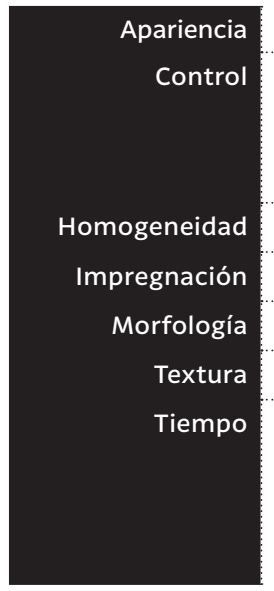

Aspecto visual del material compuesto una vez curado.

a. Control de la fibra: dominio en la manipulación de la fibra durante la preparación del compuesto.

b. Control del compuesto: dominio en la manipulación del compuesto al conformar una pieza.

Uniformidad en la composición de la mezcla de los componentes.

Absorción de la resina sobre la fibra.

Fidelidad de la forma.

Sensación al tacto, una vez curado el material compuesto.

a. Tiempo de preparación de la fibra: minutos invertidos en el proceso de corte y tamizado.

b. Tiempo de producción de las probetas: minutos invertidos en la medición de los componentes, en la mezcla de los componentes y el vertido en los moldes.

Para el objetivo específico 2: conformación del material

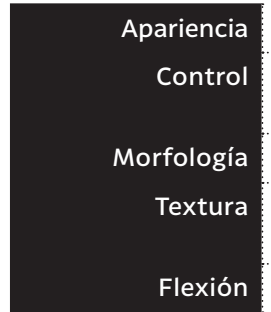

Aspecto visual del material compuesto una vez conformado.

Control del compuesto: dominio en la manipulación del compuesto al momento de conformar una pieza.

Fidelidad de la forma.

Sensación que produce al tacto el material compuesto una vez conformado.

Comportamiento del material al ser sometido a un ensayo de flexión.
Figura 2. Residuo de micro-huira. Fuente: elaboración propia. 


\subsection{Desarrollo de experimentos}

\begin{tabular}{|c|c|c|c|}
\hline $\begin{array}{l}1^{\circ} \text { Preparación } \\
\text { de la fibra }\end{array}$ & $\begin{array}{l}2^{\circ} \text { Experimentación } \\
\text { del material }\end{array}$ & $\begin{array}{l}3^{\circ} \text { Caracterización del } \\
\text { material }\end{array}$ & $\begin{array}{l}4^{\circ} \text { Conformación del } \\
\text { material }\end{array}$ \\
\hline $\begin{array}{l}\text { Chipeado de la fibra } \\
\text { Molido de la fibra } \\
\text { Tamizado de la fibra }\end{array}$ & $\begin{array}{l}\text { Prueba de adhesividad PU Khel } \\
\text { Prueba de adhesividad PU Graflex } \\
\text { Set experimental }\end{array}$ & $\begin{array}{l}\text { Ensayo mecánico de flexión } 1 \\
\text { Ensayo mecánico de flexión } 2 \\
\text { Experimentación de mecanizado } \\
\text { Ensayo de corte y grabado láser } \\
\text { Ensayo teñido de la fibra }\end{array}$ & $\begin{array}{l}\text { Conformación de doble curvatura } \\
\text { Conformación acanalada } \\
\text { Conformación laminar } \\
\text { Conformación mimbre blanco y cocido } \\
\text { Conformación con molde de ABS }\end{array}$ \\
\hline
\end{tabular}

Esquema 5. Proceso de experimentación. Esquema 6. Variables del set experimental. Fuente: elaboración propia.
Para el desarrollo de la etapa se realiza un set experimental utilizando cinco adhesivos, tres granulometrías de fibra y tres proporciones de fibra-adhesivo, con el fin de obtener probetas de $120 \times 50 \mathrm{~mm}$ de área, $9 \mathrm{~mm}$ de espesor y una densidad de $0,6 \mathrm{gr} / \mathrm{cm}^{3}$. El proceso de curado se realiza a una temperatura constante de $80^{\circ}$ en un horno industrial, por 20 minutos. Luego, se dejan reposar a temperatura ambiente durante 24 horas, antes del desmoldeo. Se obtienen cincuenta y una probetas, que son evaluadas de acuerdo a los criterios establecidos, y se evalúan según puntuación de 1 al 3, en la que es I la peor evaluación y 3, la mejor. A partir de ello, cada probeta obtiene un puntaje, las de mayor puntuación son las seleccionadas para ser sometidas a prueba mecánica de flexión.

\subsection{Caracterización del material}

6.4.1. Ensayos mecánicos de flexión. Primero, se ensayan veinte probetas que corresponden a cuatro mezclas distintas del material compuesto, son consideradas las mezclas mejor evaluadas en la etapa anterior. Para ello, se utilizó PU Khel y PU Espuma Khel, en proporción $75 \%-25 \%$, con una densidad estimada de $0,6 \mathrm{gr} / \mathrm{cm}^{3}$. De este ensayo, se selecciona una mezcla única, para un nuevo ensayo de flexión.

Para ello, se ensaya un set de veintisiete probetas, que corresponde a esta mezcla única, se utiliza PU Khel en proporción de $80 \%-20 \%$ y con densidad o,6 gr/cm³. Para la elaboración de las probetas, el tiempo y temperatura de curado se mantienen constante en 20 minutos y $80^{\circ} \mathrm{C}$; las cuales fueron realizadas gracias a la colaboración del Laboratorio de Materiales de la Facultad de Arquitectura y Urbanismo de la Universidad de Chile y los ensayos gracias a la colaboración del Laboratorio PROTEN de la UTEM.

6.4.2. Experimentación de mecanizado. Se realiza un ensayo de mecanizado al material compuesto para evaluar: a. perforación, b. corte y c. desbaste. Las pruebas se realizan sobre probetas de mimbre conformado, osb y mdf, de $9 \mathrm{~mm}$ de espesor, para realizar un análisis comparativo de sus comportamientos.

6.4.3. Ensayo de corte y grabado láser. Se ensaya una probeta laminar de 5 $\mathrm{mm}$, para evaluar el comportamiento del material compuesto al ser sometido a corte y grabado láser.

6.4.4. Ensayo teñido de la fibra. Evaluación de la absorción del mimbre desfibrado, al ser sometido y sumergido en anilina al agua. Para realizar el teñido se vierten $100 \mathrm{gr}$ de fibra de mimbre en 1 litro de agua tibia con $5 \mathrm{gr}$ de anilina diluida. Se deja remojar por 10 minutos y se seca al horno a $80^{\circ}$, por 20 minutos. 

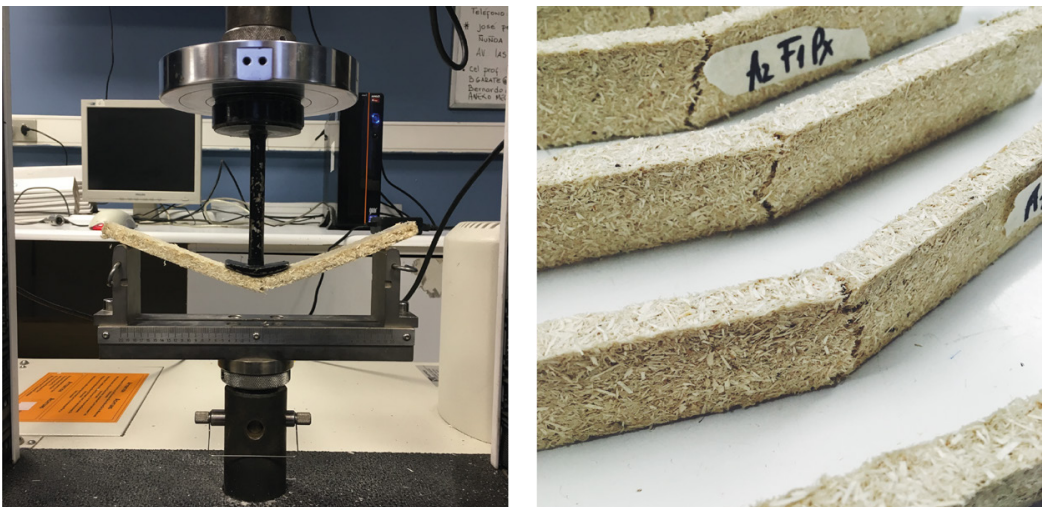

\begin{tabular}{|ccccc}
\hline PROBETA & a & b & c & d \\
\hline Tipo de adhesivo & PU Khel & PU Khel & PU Espuma Khel & PU Espuma Khel \\
\hline Granulometría & Fino & Medio & Fino & Medio \\
\hline Fibra-adhesivo & $75 \%-25 \%$ & $75 \%-25 \%$ & $75 \%-25 \%$ & $75 \%-25 \%$ \\
\hline
\end{tabular}

\subsection{Conformación del material}

En esta etapa se realizaron cinco tipos de experimentos:

1. Conformación de doble curvatura. Prueba de doble curvatura con molde machihembrado de madera terciada, que acoge un volumen semiesférico de $70 \mathrm{~mm}$ de radio exterior, para conformar una probeta de $15 \mathrm{~mm}$ de espesor, tipo cáscara o shell.

2. Conformación acanalada. Prueba para obtener una lámina acanalada, que otorgue textura y flexibilidad al material, a partir de un molde de madera e impresión 3D.

3. Conformación laminar. Prueba de conformación tipo laminar de espesor 5 $\mathrm{mm}$, para evaluar la flexibilidad y resistencia del material. Para la conformación de las probetas se utiliza un molde de MDF, dimensionado por corte láser.

4. Conformación mimbre blanco y cocido. Prueba que busca evaluar el comportamiento de la unión de las fibras y sus cualidades estéticas.

5. Conformación con molde de ABS. Se desarrolla un molde en ABS por medio de impresión 3D, para validar la conformación del material a partir de esta tecnología. Se establece un volumen de geometría simple, con un ángulo de inclinación que permita el desmolde.

Para la elaboración de las probetas, el tiempo y temperatura de curado se mantienen constantes en 20 minutos y $80^{\circ}$.

\section{Aplicación de diseño: desarrollo de una serie corta de packaging secundario 7.1. Contexto de la aplicación}

Para determinar la aplicación de diseño del material, se realizó un levantamiento de información en torno a la actividad económica de la provincia de Colchagua, lo que permitió identificar veintiocho empresas y proyectos de carácter privado y/o asociativo.

A partir de esto, y al considerar el desarrollo de la economía local, se toma como caso de estudio la Cooperativa de Berries de Chimbarongo, por su asociatividad en torno a la agricultura familiar campesina y la promoción de productos de elaboración local y de baja escala. Y a su vez, porque buscan orientar sus procesos
Figura 3. Ensayo de flexión.

Fuente: elaboración propia.

Tabla 3. Probetas para ensayo de flexión 1. 


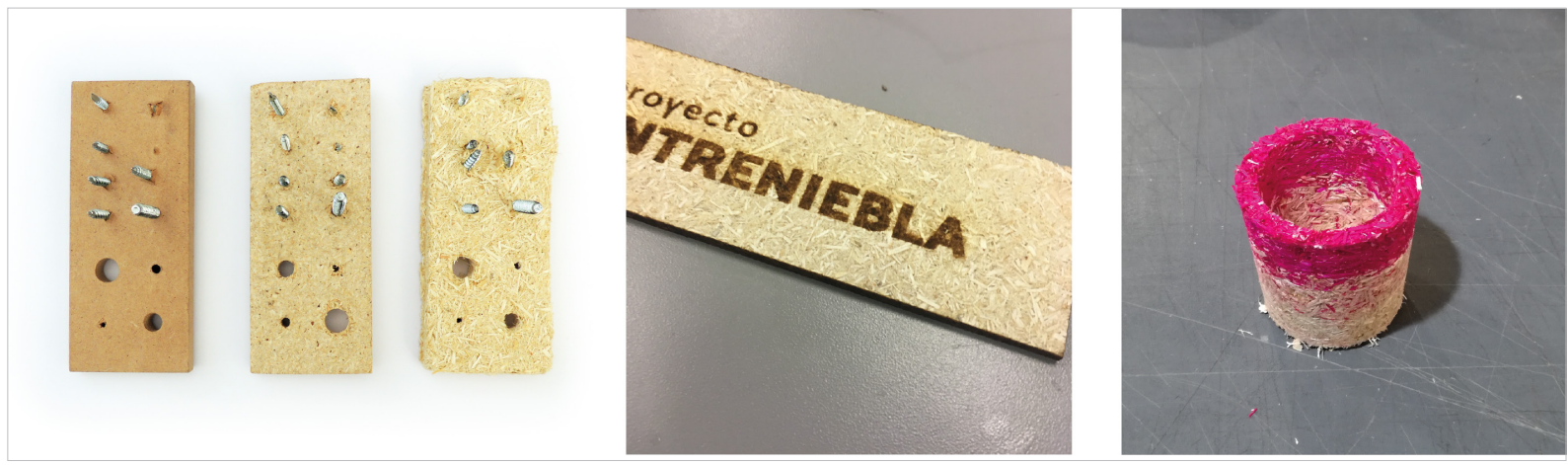

Figura 4. Prueba perforación, grabado láser y teñido. Fuente: elaboración propia. hacia la innovación, que se da por un lado a nivel de huerto, y por otro, a nivel de producto, donde buscan desarrollar preparaciones gourmety de alto valor agregado, en colaboración de profesionales del área gastronómica y diseñadores. El Proyecto EntreNiebla decide desarrollar packaging secundario que contenga el envase de vidrio de las mermeladas, escabeches, siropes y salsas para carnes; y a su vez, rediseñar las etiquetas, con el fin de lograr un producto coherente en su totalidad.

\subsection{Estado del arte}

Esta actividad consistió en buscar referentes en torno a: a. empaques de frutos rojos y mermeladas, b. empaques fabricados en madera debido a la similitud que posee con el material desarrollado, y c. productos de biocomposites y productos fabricados en matricería.

\subsection{Brief de diseño}

Desarrollar packaging secundario para los productos de Ana Córdova, presidenta de la Cooperativa de Berries.

Considerar un contenedor individual y uno doble, que tenga un segundo uso. Formatos de venta de los productos: 260 y $470 \mathrm{gr}$.

Foco: mercado gourmet.

\subsection{Desarrollo morfológico y proceso productivo}

Al considerar las propiedades mecánicas del material y el peso de los frascos del producto, se desarrollan propuestas para dos opciones de venta: contenedor doble para frascos de 260 gr y contenedor unitario para frascos de 470 gr. Para ello, se trabajan geometrías primitivas de curvatura simple y se elaboran prototipos rápidos de las propuestas utilizando corte láser de MDF, para hacer una evaluación preliminar del uso.

Para la fabricación de los contenedores se realizan moldes (en router CNC) de tres piezas: macho, hembra y tapa. Se utiliza un espesor de pared de 12 $\mathrm{mm}$ y en el modelo $3 \mathrm{D}$ se da una tolerancia de $1 \mathrm{~mm}$, lo que corresponde al espesor total del PAI que se utiliza para desmoldar.

Para el proceso de conformado se realizan los cálculos de fibra-adhesivo, donde la masa se obtiene a partir del volumen del modelo 3D, con el fin de obtener el volumen exacto que se utilizará para la conformación de la pieza y evitar mermas. El material compuesto se prensa e ingresa al horno para un curado de 20 minutos a $80^{\circ} \mathrm{C}$. Para obtener piezas más firmes, se deja enfriar el molde durante un día antes de desmoldar. 

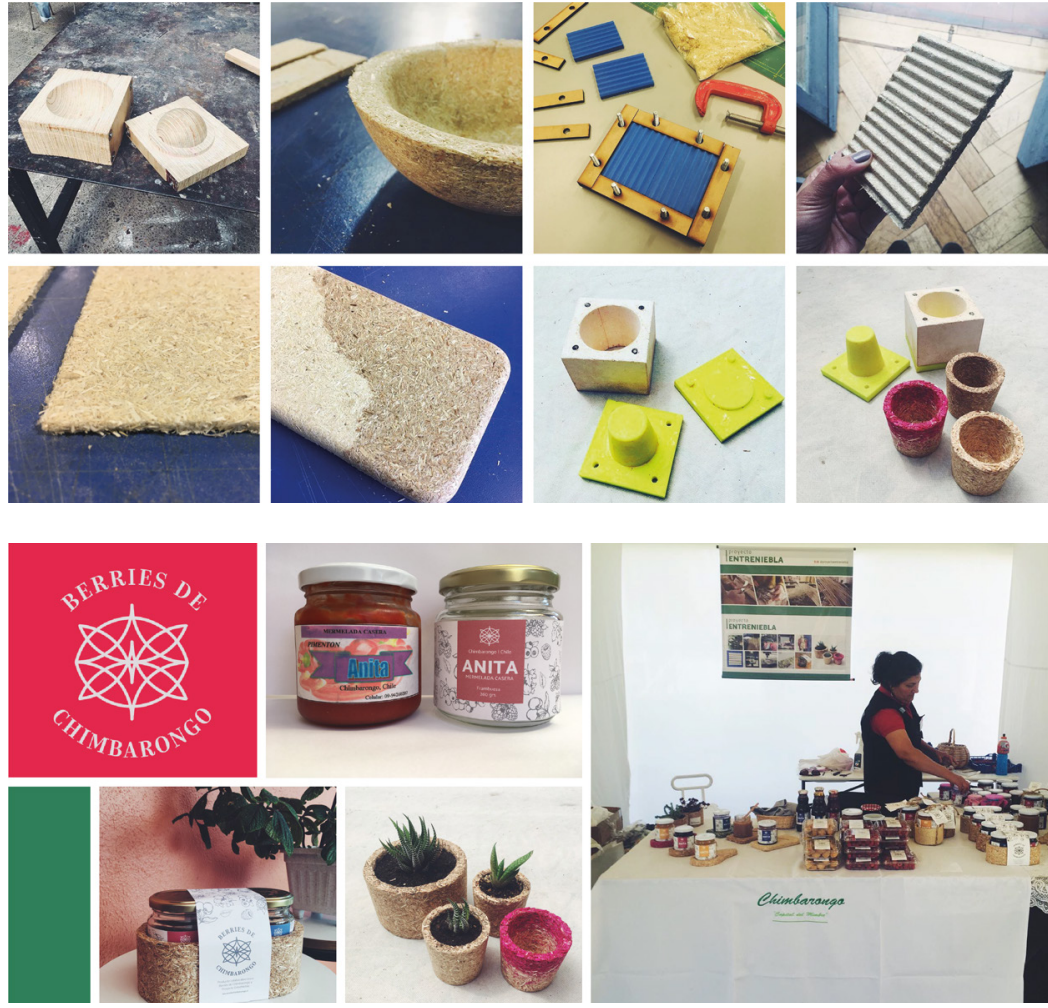

\section{Discusión de resultados}

\subsection{De la fibra}

\section{Obtención de la materia prima}

La ejecución del modelo piloto de gestión de residuos permitió visualizar el volumen producido por nueve artesanos. A partir de esto, se observa que el residuo de huira de mimbre es voluminoso y liviano, y, por el contrario, el residuo del despunte de varilla es de volumen reducido y pesado. Se considera, por lo tanto, chipear la materia prima rescatada, antes de ser transportada, para optimizar costos logísticos.

\section{Obtención de la granulometría}

A partir del proceso de obtención de la granulometría, se establece que:

a. El proceso de chipear y moler la materia prima es lento dada la tecnología a disposición, por lo que, para optimizar el proceso de obtención de la fibra, se deben tener máquinas que permitan triturar mayor cubicación por hora/hombre.

b. El proceso de tamizado debe estar enfocado en eliminar las fibras mayores a $5 \mathrm{~mm}$ de longitud, para que puedan ser trituradas nuevamente e incorporarse en la granulometría final.

A partir de la evaluación del set experimental, se establece que:

a. La granulometría $\mathrm{F}_{3}$ / 0,1 mm - $10 \mathrm{~mm}$ no cumple los criterios de morfología, impregnación, ni homogeneidad. Esto se debe a que las fibras más grandes no absorben el adhesivo, lo que genera una mala distribución de los componentes del material compuesto.
Figura 5. Experimentación formal y sus moldes. Fuente: elaboración propia.

Figura 6. Logo Berries Chimbarongo; etiqueta de Ana Córdova y etiqueta rediseñada; packaging de mimbre conformado; contenedores de mimbre; Expo Mimbre 2018. Fuente: elaboración propia. 
b. La granulometría F2 / 0,1 mm - $5 \mathrm{~mm}$ obtiene mejores resultados con respecto a la anterior, sin embargo, la distribución de la fibra mantiene espacios con aire, lo que genera que el material sea poco resistente.

c. La granulometría $\mathrm{F}_{1} / \mathrm{0}, 1 \mathrm{~mm}-3 \mathrm{~mm}$ obtiene buenos resultados en los criterios de morfología, impregnación y homogeneidad. Esto se debe a que el mimbre que se encuentra más desfibrado y poroso absorbe mejor el adhesivo, por lo que la impregnación de la fibra permite que el material compuesto se vea y sienta más homogéneo.

d. A partir de la evaluación de las 3 granulometrías se establece una combinación entre la $\mathrm{F}_{1}$ y la F2, con el fin de complementar sus buenos resultados.

\subsection{Del material biobasado}

Desempeño de los adhesivos

A partir de la evaluación de set experimental, se establece que:

a. Los adhesivos KHEL obtienen mejores resultados que el resto de los adhesivos, ya que, en la etapa de mezcla presenta buena impregnación y adherencia entre los componentes. Esta primera etapa resulta fundamental, ya que la impregnación de las fibras mejora la manipulación del compuesto, y, por lo tanto, permite un proceso de moldeo eficiente y con mejores resultados.

b. El adhesivo PVA presenta resultados buenos, pero no los esperados, ya que los criterios de homogeneidad e impregnación se ven desfavorecidos por la manipulación del adhesivo. Esto se debe a las proporciones utilizadas, las que generaron un aglutinamiento inmediato del adhesivo con la fibra de mimbre, lo que dificultó el control de la mezcla.

c. Los adhesivos Graflex no logran los resultados esperados, lo que se debe a que: no se impregnan, ni adhieren a las fibras de mimbre, resultando un material débil y quebradizo; y el adhesivo de características acuosas propicia la aparición de hongos en la fibra.

\section{Pruebas de mecanizado:}

El material permite la inserción de elementos de unión mecánica como tornillos y tarugos, sin embargo, hay desfibramiento. En cuanto al lijado, al igual que otros materiales que contienen plástico, se observa una coloración blanca en el sector tratado.

\section{Prueba de corte y grabado láser:}

El material permite ser trabajado con configuraciones de potencia y velocidad similares a los que se utilizan con el tablero de partículas. Al ser de baja densidad se corta y graba con facilidad. Sin embargo, no se obtiene un corte limpio pues el láser no logra atravesar algunas fibras.

Un problema podría radicar en la composición del material, al tener poliuretano (en muy baja concentración), el cual interactúa con el $\mathrm{CO}_{2}$ liberando hidrógeno de cianuro, gas altamente peligroso para la salud humana.

\subsection{Del molde}

Para fines de esta investigación, los moldes fabricados en madera con geometría primitiva tuvieron buenos resultados, ya que permitieron producir la serie corta y obtener resultados similares en todas las piezas.

Su fabricación no tiene un costo elevado en cuanto al material, no así el maquinado; y se estima que cada uno permitirían la reproducción de ochenta piezas antes de perder sus propiedades. 
Para desmoldar se utilizó una lámina de PAI termoformada sobre el molde. Esta no se funde con la temperatura del curado y ayuda a lograr una superficie lisa en los productos. Sin embargo, en algunas áreas donde el PAl se estiró más de lo estimado, se vuelve frágil y debe ser manipulado cuidadosamente. Además de usar desmoldante por si en algunas partes de la mezcla hay una concentración muy alta de adhesivo. Esta fragilidad hace que la lámina se deba reemplazar cada 5-10 reproducciones.

Los moldes fabricados en impresión 3D presentaron buenos resultados, dado que la temperatura de fundición del ABS se acerca a los $230^{\circ} \mathrm{C}$ (muy por sobre la temperatura de curado), por lo tanto, no sufrieron deformación por calor. Los objetos tenían geometría por revolución, por lo que queda pendiente profundizar la exploración formal que permite esta tecnología.

\section{Compuesto de residuo de mimbre y poliuretano basado en aceite de ricino} Propiedades físicas

Densidad $492,66 \mathrm{Kgf} / \mathrm{m}_{3}+/-22,82$

Propiedades mecánicas

Esfuerzo flector promedio

Módulo flector promedio

Esfuerzo de tensión

1,66 MPa +/- 0,81

$0,173 \mathrm{GPa}+/-0,050$

$1.49+/-0,56 \mathrm{MPa}$

\section{Conclusiones}

En primer lugar, el trabajo de campo permitió generar datos cuantitativos que respaldan la pertinencia del proyecto y el impacto que tendría el diseño e implementación de un plan de gestión de residuos en la comuna de Chimbarongo. Junto a ello, la base de datos de artesanos y la caracterización de esta actividad son insumos que sirven como punto de partida para futuras investigaciones enfocadas en la artesanía en mimbre.

En segundo lugar, del desarrollo experimental se obtuvieron los parámetros base para la elaboración del material, sin embargo, se desea profundizar la caracterización en torno a la humedad, resistencia al fuego, aislación acústica y aislación térmica; ya que la prueba de flexión solo permitió identificar que el comportamiento mecánico del material se acerca al de maderas naturales. Respecto a la etapa de conformación, que contempló el llenado y prensado manual de moldes de madera, se logró perfeccionar la técnica para obtener piezas firmes, con buen acabado superficial. No obstante, para el desarrollo futuro del proyecto, se propone la utilización de moldes fabricados en aluminio para extender su vida útil, entre otras ventajas, y el uso de una prensa hidráulica para la compresión, ya que se logra repartir mejor la carga, para que la densidad de la pieza obtenida sea uniforme.

Acerca de esto mismo, es necesario extender la exploración formal, con el fin de llevar al límite las capacidades del material y de los moldes, para expander sus posibles aplicaciones para el Diseño Industrial y la artesanía. Dichas aplicaciones se plantean en el ámbito de lo objetual, en productos que logren integrar el mimbre conformado con el mimbre tejido, trabajando en conjunto con artesanos de Chimbarongo para crear y fortalecer la relación diseñador-artesano, y diversificar la oferta actual de los artesanos mimbreros de la localidad. 


\section{Referencias}

Baillie, C. (ed.) 2004. Green Composites, Polymer Composites and the environment. Cambridge: Woodhead Publishing Limited.

Çöpür, Y., Güler, C., Akgül, M. \& Tapçioðlu, C. 2007. Some chemical properties of hazelnut husk and its suitability for particleboard production. Building and Environment, 42, 2568-2572.

Frihart, C. R. 2005. Wood adhesion and adhesives. In: Rowell, R. M. (ed.) Handbook of wood chemistry and wood composites. Boca Raton: CRC Press

Gay, D. (2015). Composite materials (3rd ed., p. 3). Florida: CRS Press.

Muñoz, Guillermo. (2016) Rediseño del partidor de la vara de mimbre. Santiago, Chile.

NetComposites, Materials KTN. (2014). Biocomposites: Technology overview. Retrieved from http://netcomposites.com/media/1211/biocomposites-guide.pdf

Olivares, F. (1995) Manual de diseño en plásticos reforzados con fibra de vidrio. Santiago: Ediciones Universidad Tecnológica Metropolitana.

Peek, N. (2010). Rapid prototyping of green composites. Massachusetts Institute of Technology.

Verma, D., Jain, S., Zhang, X., \& Gope, P. (2016). Green approaches to biocomposite materials science and engineering. Hershey, PA.

Wechsler, A. (2013). Sustainable Particleboards: Renewable Building Materials from Agricultural and Forestry By-products (Doctor of Philosophy). UNSW.

Wool, R \& Sun, Susan. (2018). Bio-Based Polymers and Composites / R.P. Wool, X.S. Sun. 